\title{
Ion-Specific Influence of Electrolytes on Bubble Coalescence in Nonaqueous Solvents
}

\author{
Christine L. Henry and Vincent S. J. Craig* \\ Department of Applied Mathematics, Research School of Physical Sciences and \\ Engineering, The Australian National University, Canberra ACT 0200 Australia
}

Received March 20, 2008

\begin{abstract}
We report the effects of electrolytes on bubble coalescence in nonaqueous solvents methanol, formamide, propylene carbonate, and dimethylsulfoxide (DMSO). Results in these solvents are compared to the ion-specific bubble coalescence inhibition observed in aqueous electrolyte solutions, which is predicted by simple, empirical ion combining rules. Coalescence inhibition by electrolytes is observed in all solvents, at a lower concentration range $(0.01 \mathrm{M}$ to $0.1 \mathrm{M})$ to that observed in water. Formamide shows ion-specific salt effects dependent upon ion combinations in a way analogous to the combining rules observed in water. Bubble coalescence in propylene carbonate is also consistent with ion-combining rules, but the ion assignments differ to those for water. In both methanol and DMSO all salts used are found to inhibit bubble coalescence. Our results show that electrolytes influence bubble coalescence in a rich and complex way, but with notable similarities across all solvents tested. Coalescence is influenced by the drainage of fluid between two bubbles to form a film and then the rupture of the film and one might expect that these processes will vary dramatically between solvents. The similarities in behavior we observe show that coalescence inhibition is unlikely to be related to the surface forces present but is perhaps related to the dynamic thinning and rupture of the liquid film through the hydrodynamic boundary condition.
\end{abstract}

\section{Introduction}

In the 19th century Hofmeister described the relative ability of electrolytes to precipitate proteins, and ordered the salting out strength of the anions in the well-known Hofmeister series (for an English translation see Kunz et al. ${ }^{1}$ ). Similarly the lyotropic series describes the relevant strength of cations in specific-ion phenomena. ${ }^{2}$ Since then a vast number of experiments have reported specific-ion effects whereby the behavior of a system is strongly dependent on the type of electrolyte employed. Specific-ion effects are crucial in biological systems as well as many commercial polymer and surfactant preparations. ${ }^{3}$ Despite the obvious importance of the topic, an adequate predictive theoretical description of specific-ion effects has not yet been produced, although there has been significant progress in recent years. ${ }^{4-8}$ Perhaps the simplest system described to date that exhibits specific-ion effects is the inhibition of bubble coalescence by electrolytes in water. ${ }^{9-15}$

Electrolyte Inhibition of Bubble Coalescence in Aqueous Solutions. It has been known for 100 years that some electrolytes can inhibit bubble coalescence in water at concentrations of $\sim 0.1$

* To whom correspondence should be addressed. E-mail: Vince.Craig@ anu.edu.au.

(1) Kunz, W.; Henle, J.; Ninham, B. W. Curr. Opin. Colloid Interface Sci. 2004, 9,19 .

(2) Melander, W.; Horvath, C. Arch. Biochem. Biophys. 1977, 183, 200.

(3) Kunz, W. Pure Appl. Chem. 2006, 78, 1611.

(4) Boström, M.; Deniz, V.; Frank, G. V.; Ninham, B. W. Adv. Colloid Interface Sci. 2006, 123,5 .

(5) Boström, M.; Tavares, F. W.; Bratko, D.; Ninham, B. W. J. Phys. Chem. B 2005, 109, 24489 .

(6) Jungwirth, P.; Tobias, D. J. Chem. Rev. 2006, 106, 1259.

(7) Collins, K. D. Methods 2004, 34, 300.

(8) Boström, M.; Williams, D. R. M.; Ninham, B. W. Phys. Rev. Lett. 2001, 87,8103 .

(9) Marrucci, G.; Nicodemo, L. Chem. Eng. Sci. 1967, 22, 1257.

(10) Lee, J. C.; Hodgson, T. D. Chem. Eng. Sci. 1968, 23, 1375.

(11) Lessard, R. R.; Zieminski, S. A. Ind. Eng. Chem. Fundam. 1971, 10, 260.

(12) Sagert, N. H.; Quinn, M. J. Chem. Eng. Sci. 1977, 33, 1087.

(13) Oolman, T. O.; Blanch, H. W. Chem. Eng. Comms 1986, 43, 237.

(14) Prince, M. J.; Blanch, H. W. AIChE J. 1990, 36, 1425.

(15) Deschenes, L. A.; Barrett, J.; Muller, L. J.; Fourkas, J. T.; Mohanty, U. J. Phys. Chem. B 1998, 102, 5115.
Table 1. Combining Rules for Effects on Bubble Coalescence Inhibition of Selected Salts in Water ${ }^{a}$

\begin{tabular}{lllllllllll}
\hline \multicolumn{1}{c}{ ion } & & $\mathrm{Ca}^{2+}$ & $\mathrm{Mg}^{2+}$ & $\mathrm{H}^{+}$ & $\mathrm{Li}^{+}$ & $\mathrm{Na}^{+}$ & $\mathrm{K}^{+}$ & $\mathrm{NH}_{4}^{+}$ & $\left(\mathrm{CH}_{3}\right)_{4} \mathrm{~N}^{+}$ \\
\hline & assignment & $\alpha$ & $\alpha$ & $\beta$ & $\alpha$ & $\alpha$ & $\alpha$ & $\alpha$ & $\beta$ \\
$\mathrm{SCN}^{-}$ & $\beta$ & & & & & $\mathrm{x}$ & & $\mathrm{x}$ & \\
$\mathrm{ClO}_{4-}^{-}$ & $\beta$ & & $\mathrm{x}$ & $\checkmark$ & & $\mathrm{x}$ & & $\mathrm{x}$ & \\
$\mathrm{I}^{-}{ }^{-}$ & $\alpha$ & & & & $\checkmark$ & $\checkmark$ & $\checkmark$ & & \\
$\mathrm{ClO}_{3}{ }^{-}$ & $\beta$ & & & & & $\mathrm{x}$ & & & \\
$\mathrm{NO}_{3}{ }^{-}$ & $\alpha$ & $\checkmark$ & & $\mathrm{x}$ & $\checkmark$ & $\checkmark$ & $\checkmark$ & $\checkmark$ & \\
$\mathrm{Br}^{-}$ & $\alpha$ & & & $\mathrm{x}$ & & $\checkmark$ & $\checkmark$ & & $\mathrm{x}$ \\
$\mathrm{Cl}^{-}$ & $\alpha$ & $\checkmark$ & $\checkmark$ & $\mathrm{x}$ & $\checkmark$ & $\checkmark$ & $\checkmark$ & $\checkmark$ & $\mathrm{x}$ \\
$\mathrm{CH}_{3} \mathrm{COO}^{-}$ & $\beta$ & & $\mathrm{x}$ & $\checkmark$ & & $\mathrm{x}$ & $\mathrm{x}$ & $\mathrm{x}$ & $\checkmark$ \\
$\mathrm{SO}_{4}{ }^{-2}$ & $\alpha$ & & $\checkmark$ & $\mathrm{x}$ & $\checkmark$ & $\checkmark$ & $\checkmark$ & &
\end{tabular}

${ }^{a}$ Adapted and extended from ref $18 .^{b}(\checkmark)$ Indicates bubble coalescence inhibition; $\alpha \alpha$ and $\beta \beta$ salts. (x) Indicates no inhibition relative to pure water; $\alpha \beta$ and $\beta \alpha$ salts.

M. ${ }^{11,16,17}$ An example of this effect is the foamy whitewater that is created when waves crash at the beach. This effect has also been employed to promote flotation of naturally hydrophobic minerals. Notably, some electrolytes at concentrations up to 0.5 $\mathrm{M}$ have little or no effect on bubble coalescence. The coalescence inhibition is dependent upon the identities of the ions present. It was demonstrated that by empirically assigning ions a value of either $\alpha$ or $\beta$ an electrolyte's coalescence inhibition behavior could be described using simple combining rules. ${ }^{18,19}$ An electrolyte formed from an $\alpha$ cation and an $\alpha$ anion will inhibit bubble coalescence as will an electrolyte formed from a $\beta$ cation and $\beta$ anion. However electrolytes formed from an $\alpha$ cation and a $\beta$ anion or $\beta$ cation and $\alpha$ cation will have no effect. This is more easily understood by referring to Table 1 , where $\alpha \alpha$ or $\beta \beta$ result in a bubble coalescence inhibition (indicated by a tick) and $\alpha \beta$ or $\beta \alpha$ result in no inhibition (as indicated by a cross). We have not found any exceptions to these simple predictive rules. Currently it is unclear precisely what properties makes

(16) Foulk, C. W.; Miller, J. N. Ind. Eng. Chem. 1931, 23, 1283.

(17) Pollock, J. A. Philos. Mag. 1912, 24, 189.

(18) Craig, V. S. J.; Ninham, B. W.; Pashley, R. M. J. Phys. Chem. 1993, 97, 10192.

(19) Craig, V. S. J.; Ninham, B. W.; Pashley, R. M. Nature 1993, 364, 317. 
an ion an $\alpha$ or $\beta$ ion and it is an aim of this study to elucidate this.

Notwithstanding the simplicity of these rules, a good understanding of the chemistry and physics at play in bubble coalescence inhibition remains elusive. Thermodynamically, the introduction of bubbles (i.e., the creation of interface) in a pure liquid is unfavorable, and therefore bubbles colliding should rapidly coalesce to minimize the interfacial area and the energy of the system. In pure liquid there is no stabilization mechanism by which this coalescence can be opposed beyond hydrodynamic repulsion, ${ }^{20}$ whereas in solutions the presence of a solute leads to a Gibbs elasticity of the interface. ${ }^{21}$ Under other stabilizing mechanisms such as Gibbs-Marangoni flows, alteration of the hydrodynamic boundary conditions to those of no-slip and repulsive surface forces can also arise, depending on the nature of the solute. Whereas the stabilizing mechanisms at play in surfactant solutions are well studied and adequately described, the mechanisms operating in aqueous electrolyte solutions remain unclear. ${ }^{22,23}$ We note that bubble coalescence still occurs but it takes longer than in pure solution with the bubble lifetime at an interface typically being extended from less than a second to $\sim 5$ $\mathrm{s}$ for a bubble rising to a free interface. ${ }^{24} \mathrm{We}$ have proposed that some electrolytes may act to alter the hydrodynamic boundary condition and thereby extend the time required for the growth of bridging capillary waves beyond the typical collision time ${ }^{25}$ but this is yet to be tested.

In a recent publication we showed that the behavior of mixed electrolytes in water could be explained by extending the empirical combining rules developed in single electrolytes. ${ }^{25}$ Our results in mixed electrolytes were also consistent with the hypothesis put forward by Marcelja that inhibition depends upon ion separation within the interfacial region. ${ }^{26}$ Simulation $^{6,26,27}$ and spectroscopic studies ${ }^{28-34}$ have led to a general acceptance that some ions can show a surface preference within the interfacial region, while others show no such preference and exist subsurface. However, due to the necessity for distance resolution to Angstrom level at a mobile surface containing few ions, results for different species have been inconclusive and occasionally contradictory. We cannot consistently relate ion interfacial position to the influence that ions have on bubble coalescence in water, but it is of interest to investigate how microscopic solution structure at the interface may influence coalescence inhibition.

Electrolyte Inhibition of Bubble Coalescence in Nonaqueous Solutions. We here report on the influence of electrolytes on bubble coalescence in a range of nonaqueous solvents. The use

(20) Aveyard, R.; Binks, B. P.; Clint, J. H.; Fletcher, P. D. I. Foams and emulsions: Their stability and breakdown by solid particles and liquid droplets. The colloid chemistry of a dog's breakfast. In Foams and Emulsions; Sadoc, J. F., Rivier, N., Eds.; Kluwer Academic: Dordrecht, 1999; pp 21.

(21) Gibbs, J. W. The Collected Works; Longmans: Green and Co., New York, 1928; Vol. I, p 300.

(22) Craig, V. S. J. Curr. Opin. Colloid Interface Sci. 2004, 9, 178.

(23) Chan, B. S.; Tsang, Y. H. J. Colloid Interface Sci. 2005, 286, 410.

(24) Ghosh, P. Chem. Eng. Res. Des. 2004, 82, 849.

(25) Henry, C. L.; Dalton, C. N.; Scruton, L.; Craig, V. S. J. J. Phys. Chem. C 2007, 111, 1015 .

(26) Marcelja, S. J. Phys. Chem. B 2006, 110, 13062.

(27) Jungwirth, P.; Tobias, D. J. J. Phys. Chem. B 2002, 106, 6361.

(28) Ghosal, S.; Hemminger, J. C.; Bluhm, H.; Mun, B. S.; Hebenstreit, E. L. D.; Ketteler, G.; Ogletree, D. F.; Requejo, F. G.; Salmeron, M. Science 2005, 307, 563.

(29) Mucha, M.; Frigato, T.; Levering, L. M.; Allen, H. C.; Tobias, D. J.;

Dang, L. X.; Jungwirth, P. J. Phys. Chem. B 2005, 109, 7617.

(30) Petersen, P. B.; Saykally, R. J. J. Phys. Chem. B 2005, 109, 7976.

(31) Raymond, E. A.; Richmond, G. L. J. Phys. Chem. B 2004, 108, 5051.

(32) Soule, M. C. K.; Blower, P. G.; Richmond, G. L. J. Phys. Chem. A 2007, $111,3349$.

(33) Viswanath, P.; Motschmann, H. J. Phys. Chem. C 2007, 111, 4484.

(34) Vbrka, L.; Mucha, M.; Minofar, B.; Jungwirth, P.; Brown, E. C.; Tobias,

D. J. Curr. Opin. Colloid Interface Sci. 2004, 9, 67. of nonaqueous solvents allows us to vary the solvent physical properties and the chemical interaction with ions. Solvent physical properties are expected to influence bubble stability and the coalescence mechanism. The drainage is proportional to the viscosity; the boundary condition is influenced by the propensity of the solute for the interface; the magnitude of the interfacial charge and the electrostatic component of the surface forces are influenced by the dielectric constant; the electrodynamic component is governed by the absorption spectra; and finally the surface tension will influence the rate of growth of capillary waves. The chemical interaction of ions and solvent, and in particular the interfacial ion behavior, is also expected to vary between solvents and may manifest as changes in concentrationdependence or ion specificity of coalescence inhibition. We can compare our observations to recent spectroscopic and simulation interfacial studies in nonaqueous solvents. ${ }^{35}$

Thus, we aimed to determine: if electrolytes inhibit coalescence in nonaqueous solvents; if this coalescence inhibition behavior shows ion-specificity that can be rationalized by combining rules; if so, whether the empirical assignments to ions (i.e., $\alpha$ and $\beta$ ) differ in different solvents. Further, nonaqueous solution data may be a valuable addition to the general field of ion-specificity. To date ion-specific bubble coalescence and ion-specific trends observed in soft-matter systems have not been comparable, presumably due to the very different nature of the air-water interface and the oil-water or protein-water interfaces. We anticipate that bubble coalescence measurements in nonaqueous solvent systems should provide results that can be compared to previous bubble coalescence studies and at the same time bring the bubble coalescence studies a little closer to complex realworld soft matter systems that exhibit specific-ion effects.

We have investigated electrolyte inhibition of coalescence in nonaqueous solvents methanol, formamide, propylene carbonate and dimethylsulfoxide (DMSO). Ideally we would study more solvents but the choice of solvent available to us is limited by the inability of most nonaqueous solvents to dissolve a range of electrolytes at sufficiently high concentrations, as well as by issues of chemical stability and safety. Pertinent physical properties are presented in Table 2.

\section{Experimental Section}

All water used was purified using a MilliQ gradient system. The solvents propylene carbonate (anhydrous, purity 99.7\%), methanol (anhydrous, purity 99.8\%), and DMSO (anhydrous, purity 99.9\%) were used as obtained from Sigma Aldrich. Formamide (>99\% purity) was used as obtained from Fluka and from BDH. Salts were roasted or freeze-dried to remove water; in some cases salts were used as received. Concentrated aqueous acids were used as received, as were methanolic $\mathrm{HCl}$ (Sigma Aldrich) and methanolic $\mathrm{H}_{2} \mathrm{SO}_{4}$ (Sigma Aldrich). Propylene carbonate and DMSO stock solutions were made up under $\mathrm{N}_{2}$ in a glovebag. Methanol and formamide stock solutions were made up in a fumehood with minimal exposure to air. Stock solutions varied between 0.15 and $1 \mathrm{M}$ depending on the solubility of the salt being investigated. Bubble coalescence showed no dependence on the stock concentration used.

The experimental arrangement is depicted in Figure 1. In each experiment a quantity of pure solvent $(41.0 \mathrm{~mL})$ was placed into a cylindrical glass tube with a sintered glass frit at the bottom and a constriction some $30 \mathrm{~mm}$ above the frit. A stable flow of $\mathrm{N}_{2}$ gas (12 $\mathrm{mL} / \mathrm{s}$ ) into the tube through the frit results in a bubble stream rising in the column. A concentrated electrolyte solution is then titrated into the pure solvent. A beam of light from a diode laser is expanded and passed through the bubbling solvent before being condensed onto a photodiode detector. When coalescence is readily taking place

(35) Cwiklik, L.; Andersson, G.; Dang, L. X.; Jungwirth, P. Chem. Phys. Chem. 2007, 8, 1457. 
Table 2. Comparison of Solvent Properties ${ }^{a}$

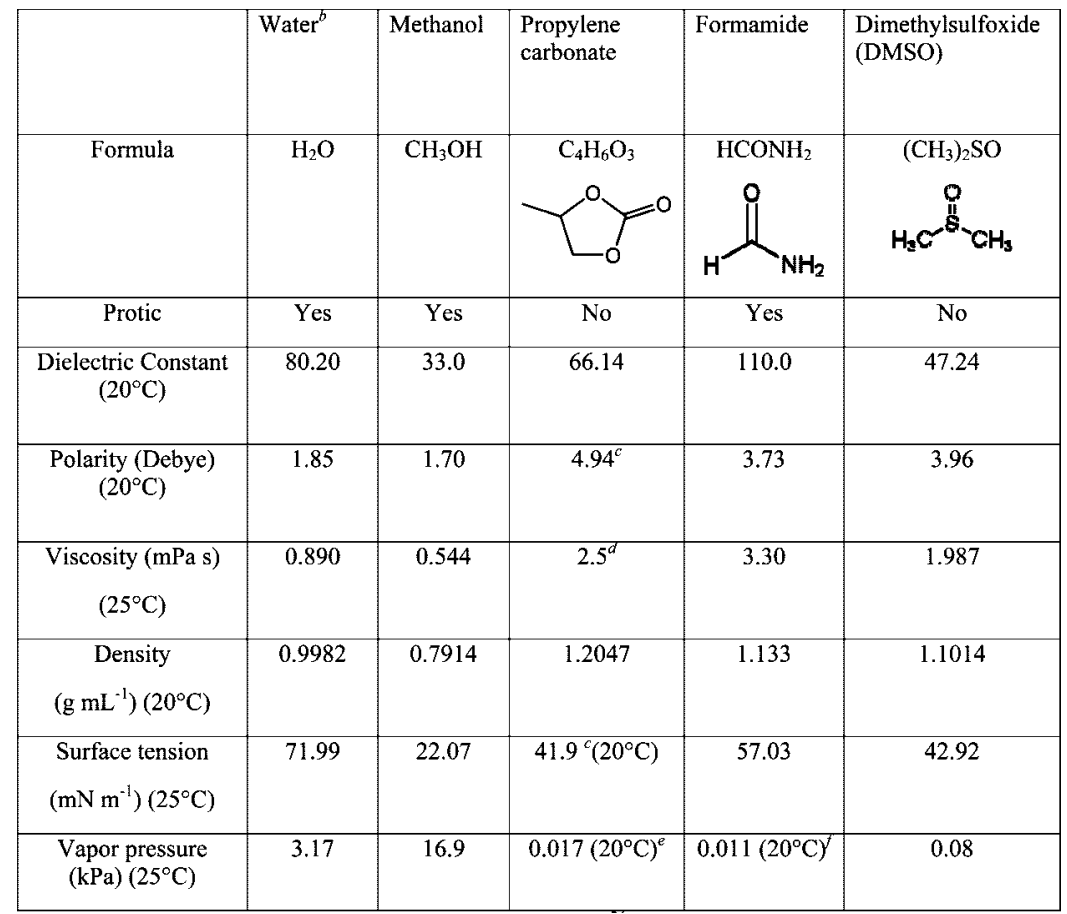

\footnotetext{
${ }^{a}$ Data taken from Handbook of Chemistry and Physics ${ }^{36}$ except where otherwise referenced. ${ }^{b}$ Results reported in Craig et al. ${ }^{19}{ }^{c}$ Data from http://macro.lsu.edu/howto/solvents/Dipole\%20Moment.htm. ${ }^{37 d}$ Data from Barthel et al. ${ }^{38} e^{e}$ Data from MSDS. ${ }^{39 f}$ Data from MSDS. ${ }^{40}$
}

the amount of light reaching the detector is high as is the measured voltage. For pure solvent this is defined as the $100 \%$ coalescence level. As bubble coalescence inhibition occurs the small bubbles created at the frit remain small and more light is scattered, lowering the voltage at the detector. At a sufficiently high concentration of a coalescence-inhibiting electrolyte the voltage stabilizes at a minimum level. This is defined as $0 \%$ coalescence. Intermediate voltages are converted to bubble coalescence using a linear conversion. The baseline level in inhibiting electrolytes is used to determine percentage coalescence for noninhibiting or partially inhibiting salts. The apparatus has been previously described. ${ }^{18}$ However for the current work the Perspex box was made nearly airtight, and $\mathrm{N}_{2}$ gas was passed through the chamber for $\sim 1 \mathrm{~h}$ before experiments began, to minimize humidity. In some cases a vacuum line was used to extract vapor-loaded gas from the box. For some experiments a blanket of $\mathrm{N}_{2}$ gas was used to cover the top of the burette containing stock solution; alternatively the burette was covered when not in use to minimize uptake of atmospheric $\mathrm{H}_{2} \mathrm{O}$. The concern was that the presence of water as a contaminant in the solvents could influence the coalescence behavior. For all solvents employed the effect of water content on bubble coalescence was investigated, in the absence and presence of electrolytes. Results for individual solvents are discussed in more detail below. All experiments were done at room temperature $\left(\sim 23^{\circ} \mathrm{C}\right)$.

\section{Results}

Influence of Atmospheric Humidity. It was not possible to conduct the bubble coalescence experiments in a manner such that the solutions would at no stage be exposed to atmospheric humidity. Therefore tests were performed to investigate the possible influence of water on the bubble coalescence measurements. This was done by purposely adding water to the solvents and measuring changes in the light level reaching the photodiode. The sensitivity of the solvents to added water differed, but at high enough concentrations in all solvents water acts to inhibit bubble coalescence. This effect is small enough to be distinguished from the electrolyte effect. Tests in propylene carbonate and in methanol showed that the effects of water and of electrolyte are essentially additive and independent (within the sensitivity of the instrument).

Bubble coalescence in both propylene carbonate and formamide is relatively insensitive to added water. The photodiode signal was unaffected up to $\sim 5 \% \mathrm{v} / \mathrm{v}(\sim 2 \mathrm{~mL}$ in $41 \mathrm{~mL}$ of solvent). In DMSO, the effect of water becomes significant at $1.2 \%(\mathrm{v} / \mathrm{v})$, and at $\sim 5 \%(\mathrm{v} / \mathrm{v})$ water the signal had decreased from 2.5 to 2.0 $\mathrm{V}$. This corresponds to a bubble coalescence change of $25 \%$. As the level of water uptake by the solvents is expected to be well below $0.1 \%$ by volume, the contribution to the observed coalescence inhibition is expected to be very small; however, the presence of trace water levels may mean that transition concentration values for electrolytes are slightly underestimated. The values observed are stable over time (within error), indicating that uptake of atmospheric moisture is not significant.

Methanol bubble stability shows the greatest sensitivity to dissolved $\mathrm{H}_{2} \mathrm{O}$. There is an immediate decrease in signal (increase in coalescence) on addition of even $0.1 \mathrm{~mL}(\sim 0.2 \% \mathrm{v} / \mathrm{v})$ water. To observe the effect of a smaller amount of water, a solution of $1 \% \mathrm{H}_{2} \mathrm{O}$ in methanol by weight was used as stock. The final concentration of $\mathrm{H}_{2} \mathrm{O}$ was then $\sim 0.4 \%$ by weight. The observed signal was slightly lower ( $<5 \%$ coalescence) than in pure methanol. This indicates that, while water does act to inhibit bubble coalescence even at this low concentration, the effect is much less than observed when electrolytes are added, at any reasonable level of contaminating $\mathrm{H}_{2} \mathrm{O}$. We also noted that bubbling for one to two hours (the course of an experiment) in an undisturbed column led to a small decrease in photodiode voltage, or increase in bubble coalescence (5\% coalescence). This may be due to absorption of water from the atmosphere. Also, because methanol is volatile, it readily evaporated and condensed on the outside of the bubble column. This may influence the experiment in two ways: first, the concentration of contaminant (and electrolyte) will increase slightly during an 


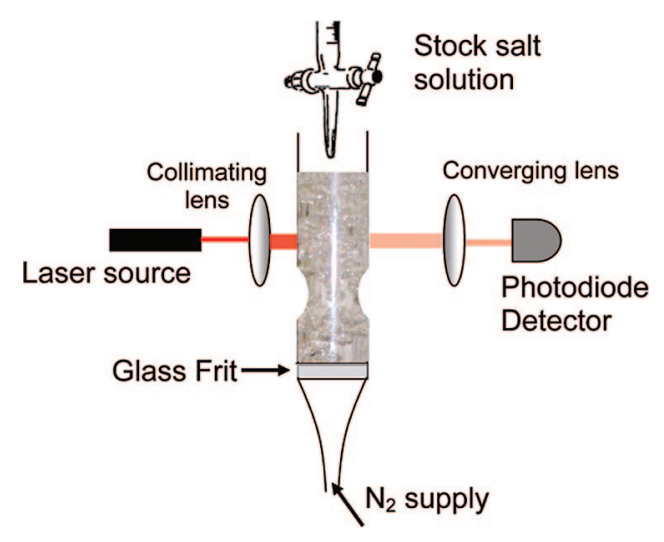

Figure 1. Bubble coalescence apparatus. Small bubbles are produced at the frit and rise in the column passing through the constriction that promotes bubble collisions. In pure solvent they rapidly coalesce such that the bubbles breaking the laser beam are large and few in number. In this case, most of the light is transmitted and strikes the detector. When coalescence is inhibited the bubbles remain small and numerous, resulting in a large amount of scattering and low light intensity at the detector.

experiment; second, the $\mathrm{N}_{2}$ atmosphere could not be maintained, as it was necessary to wipe the column to avoid scattering by external condensation, and this meant briefly opening the experiment to ambient conditions.

Methanol. Despite having the lowest dielectric constant of all the solvents studied, methanol dissolves a wide range of electrolytes. ${ }^{41}$ Thirteen different electrolytes were investigated, and all electrolytes studied were found to inhibit bubble coalescence over a relatively narrow concentration range (as seen in Figure 2). Transition concentrations (corresponding to $50 \%$ coalescence inhibition) for all electrolytes tested are reported in Table 5. These data demonstrate that the ability of electrolytes to inhibit bubble coalescence is not confined to aqueous systems. However, methanol shows little difference between salts, and none of the salts tested act as "noninhibitors". It is also noteworthy that electrolytes in methanol show no correlation between transition concentration and ionic strength, whereas a strong correlation was found among inhibiting salts in aqueous solution. ${ }^{18}$ The transition concentration for electrolytes in methanol is more than $50 \%$ lower than that typically found in water.

Formamide. The effect of electrolytes on bubble coalescence in formamide is remarkably similar to their effect in water. Some salts tested show strong inhibiting character, at concentrations similar to those seen in inhibiting salts in water. Others show no effect or need a much higher concentration to elicit an equivalent inhibition in coalescence (see Figure 3). If these latter two categories (less inhibiting and noninhibiting) are grouped, then the combining rules and designations observed in water also apply as predictors of the salt effect in formamide, for most species tested (see Table 3). Tetramethylammonium acetate, however, produces an inconsistent result: it should be (as in water) a $\beta \beta$ salt that inhibits bubble coalescence relative to pure formamide. Instead, addition of the concentrated solution to the bubble column increases photodiode signal, which generally indicates lower scattering and increased coalescence relative to the pure solvent. This belies observations that tetramethylammonium acetate in fact does act to inhibit bubble coalescence.

(36) CRC Handbook of Chemistry and Physcis, 80th ed.; CRC Press: Boca Raton, FL, 2000.

(37) http://macro.lsu.edu/howto/solvents/Dipole\%20Moment.htm.

(38) Barthel, J.; Neueder, R.; Roch, H. J. Chem. Eng. Data 2000, 45, 1007.

(39) SigmaAldrich. Propylene Carbonate MSDS No. 82226 [online], 2006.

(40) SigmaAldrich. Formamide MSDS No. F7503 [online], 2007.

(41) Stenger, V. A. J. Chem. Eng. Data 1996, 41, 1111.

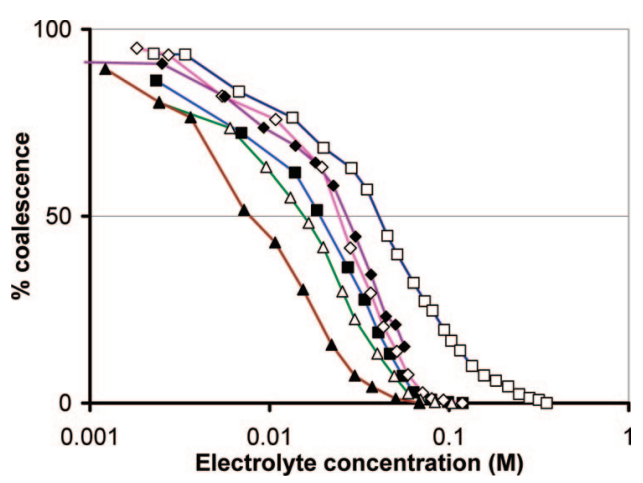

Figure 2. Inhibition of bubble coalescence by selected electrolytes in methanol solutions as a function of concentration. $\mathrm{NH}_{4} \mathrm{SCN}(\diamond), \mathrm{MgCl}_{2}$ $(\diamond), \mathrm{H}_{2} \mathrm{SO}_{4}(\square), \mathrm{NaClO}_{4}(\diamond), \mathrm{NaCl}(\diamond), \mathrm{HCl}(\square) .100 \%$ coalescence is defined in pure methanol; $0 \%$ coalescence is a stable low voltage signal in inhibiting electrolytes.

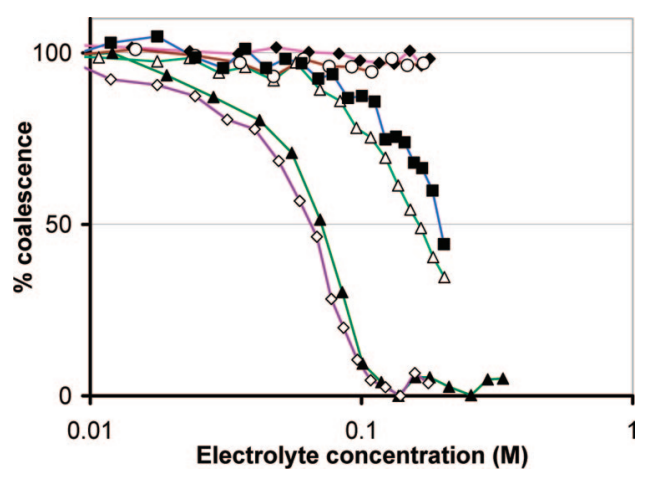

Figure 3. Inhibition of bubble coalescence by selected electrolytes in formamide solutions as a function of concentration. $\mathrm{NaI}(\diamond), \mathrm{LiCl}(\boldsymbol{\Delta})$, $\mathrm{LiClO}_{4}(\Delta),\left(\mathrm{CH}_{3}\right)_{4} \mathrm{NBr}(\boldsymbol{\square}), \mathrm{CH}_{3} \mathrm{COONa}(\bullet), \mathrm{CH}_{3} \mathrm{COONH}_{4}(\mathrm{O}) .100 \%$ coalescence is defined in pure formamide; $0 \%$ coalescence is a stable, low, voltage signal in inhibiting electrolytes.

Table 3. Ion Assignments in Formamide

\begin{tabular}{llllll}
\hline \multicolumn{1}{c}{ ions } & & $\mathrm{Li}^{+}$ & $\mathrm{Na}^{+}$ & $\mathrm{NH}_{4}^{+}$ & $\left(\mathrm{CH}_{3}\right)_{4} \mathrm{~N}^{+}$ \\
\hline $\mathrm{Cl}^{-}$ & assignment & $\alpha$ & $\alpha$ & $\alpha$ & $\beta$ \\
$\mathrm{Br}^{-}$ & $\alpha$ & $\alpha$ & & & \\
$\mathrm{I}^{-}$ & $\alpha$ & & & & $\mathrm{x}$ \\
$\mathrm{ClO}_{4}^{-}$ & $\alpha$ & $\checkmark$ & $\checkmark$ & & \\
$\mathrm{CH}_{3} \mathrm{COO}^{-}$ & $\beta$ & $\mathrm{x}$ & & & \\
& $\beta$ & & $\mathrm{xx}$ & $\mathrm{xx}$ & $\mathrm{x}^{b}$
\end{tabular}

${ }^{a}(\checkmark)$ indicates bubble coalescence inhibition. $\alpha \alpha$ and $\beta \beta$ salts. (x) and (xx) indicate partial inhibition and no inhibition, repectively, relative to pure formamide. $\alpha \beta$ and $\beta \alpha$ salts. ${ }^{b}$ Tetramethylammonium acetate is believed to be affected by contamination.

Evidence for this lies in the (qualitative) "swirl test" - a single swirl of the stock solution yields significant differences visually between inhibiting salts, which generate a slight foam that exists for several seconds after swirling, and noninhibitors such as sodium acetate, in which no long-lived bubbles are seen. Tetramethylammonium acetate is a highly hygroscopic and unstable solid, and so the results recorded are possibly influenced by contamination. As combining rules are followed we are able to summarize the coalescence inhibition in a table as previously used for water, see Table 3.

Propylene Carbonate. The influence of eight different electrolytes on bubble coalescence in propylene carbonate solutions was investigated. Electrolytes inhibit bubble coalescence in propylene carbonate, and ion specificity is observed. The four alkali metal salts tested all inhibited bubble coalescence relative to the pure solvent, over a uniform concentration range (see Figure 4). $\mathrm{HCl}$ and $\mathrm{HBr}$ (added as concentrated aqueous solutions) 


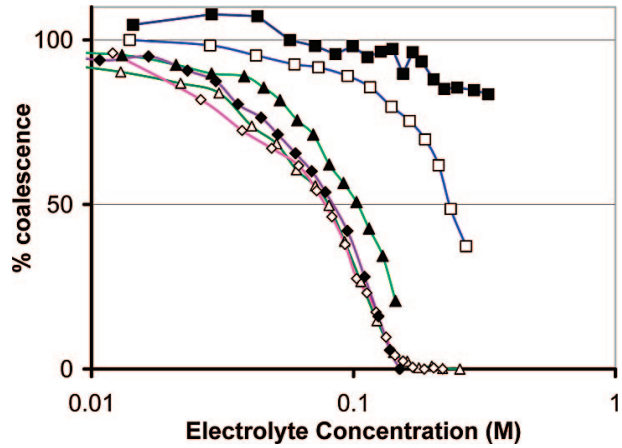

Figure 4. Inhibition of bubble coalescence by selected electrolytes in propylene carbonate solutions plotted against log of concentration. $\mathrm{NaClO}_{4}(\diamond), \mathrm{LiClO}_{4}(\Delta), \mathrm{NaSCN}(\diamond), \mathrm{LiBr}(\boldsymbol{\Delta}), \mathrm{HBr}(\square), \mathrm{HCl}(\boldsymbol{\square})$. $100 \%$ coalescence is defined in pure propylene carbonate; $0 \%$ coalescence is a stable, low, voltage signal in inhibiting electrolytes. Acids are added as concentrated aqueous solutions.

Table 4. Ion Assignments in Propylene Carbonate

\begin{tabular}{lllll}
\hline Ions & & $\mathrm{Li}^{+}$ & $\mathrm{Na}^{+}$ & $\mathrm{H}^{+}$ \\
\hline $\mathrm{Cl}^{-}$ & assignment & \\
$\mathrm{Br}^{-}$ & $\alpha$ & $\alpha$ & $\alpha$ & $\beta$ \\
$\mathrm{SCN}^{-}$ & $\alpha$ & $\checkmark$ & & $\mathrm{x}$ \\
$\mathrm{ClO}_{4}^{-}$ & $\alpha$ & & $\checkmark$ & $\mathrm{x}$ \\
( & $\alpha$ & $\checkmark$ & $\checkmark$ &
\end{tabular}

${ }^{a}(\checkmark)$ indicates bubble coalescence inhibition. $\alpha \alpha$ and $\beta \beta$ salts. (x) indicates partial inhibition relative to pure propylene carbonate. $\beta \alpha$ salts.

showed no coalescence inhibition up to significantly higher concentrations, upon which the volume of nonpropylene carbonate solvent becomes important; here, the inhibition may be largely due to water rather than electrolyte effects. Methanolic $\mathrm{HCl}$ was also used and its behavior was consistent with the aqueous acid. These acids are categorized as "less inhibiting" than other electrolytes used.

We therefore observe in propylene carbonate two groups of salts as seen in water. We can create a set of combining rules for cations and anions analogous to those seen in water, that holds for the six electrolytes used here, see Table 4. By use of comparable definitions, $\mathrm{Na}^{+}$and $\mathrm{Li}^{+}$would be $\alpha$ cations and $\mathrm{H}^{+}$ a $\beta$ cation. All anions here used $\left(\mathrm{Cl}^{-}, \mathrm{Br}^{-}, \mathrm{ClO}_{4}^{-}\right.$, and $\left.\mathrm{SCN}^{-}\right)$ would be in one class, as $\alpha$ anions. This indicates that ion assignments in nonaqueous solvents may disagree from those in water: aqueous $\mathrm{ClO}_{4}{ }^{-}$and $\mathrm{SCN}^{-}$are in a different class ( $\beta$ anions) to $\mathrm{Cl}^{-}$and $\mathrm{Br}^{-}$, and $\mathrm{NaClO}_{4}$ and $\mathrm{NaSCN}$, for example, have no effect on aqueous bubble coalescence.

DMSO. Four electrolytes were studied in DMSO, and all were found to inhibit coalescence over a narrow concentration range, as seen in Figure 5. Low solubility and high reactivity prevent a wider selection of salts being used. We note one difference between DMSO and water: sodium perchlorate does not inhibit bubble coalescence in aqueous solutions, but here it acts as an inhibiting salt.

\section{Discussion}

We have shown that partial foam stabilization by electrolytes is not unique to aqueous solutions. Methanol, formamide, propylene carbonate, and DMSO all exhibit coalescence inhibition. Furthermore, propylene carbonate and formamide show evidence of ion-specific cation/anion combining rules, as observed in water. In propylene carbonate the ion assignments to different classes vary from those in water; in formamide the same assignments as those made for water apply across the salts tested. Methanol and DMSO show little difference between electrolytes,
Table 5. Transition Concentrations for Bubble Coalescence Inhibition of Electrolytes ${ }^{a}$

\begin{tabular}{|c|c|c|c|c|c|}
\hline salt & $\mathrm{H}_{2} \mathrm{O}$ & methanol & formamide & $\begin{array}{l}\text { propylene } \\
\text { carbonate }\end{array}$ & DMSO \\
\hline $\mathrm{HCl}$ & NI & 0.04 & & NI & \\
\hline $\mathrm{HBr}$ & NI & & & NI & \\
\hline $\mathrm{H}_{2} \mathrm{SO}_{4}$ & NI & 0.019 & & & \\
\hline $\mathrm{LiCl}$ & 0.095 & & 0.072 & & \\
\hline $\mathrm{LiBr}$ & & & & 0.105 & \\
\hline LiI & 0.34 & & 0.079 & & \\
\hline $\mathrm{LiClO}_{4}$ & $\mathrm{NI}$ & & 0.16 & 0.081 & \\
\hline $\mathrm{NaCl}$ & 0.095 & 0.023 & & & \\
\hline $\mathrm{NaBr}$ & 0.13 & 0.023 & & & 0.059 \\
\hline $\mathrm{NaI}$ & 0.30 & & 0.065 & & \\
\hline $\mathrm{NaNO}_{3}$ & 0.101 & 0.025 & & & \\
\hline $\mathrm{NaClO}_{4}$ & NI & 0.024 & & 0.078 & 0.082 \\
\hline $\mathrm{NaOOCCH}_{3}$ & NI & 0.031 & NI & & \\
\hline $\mathrm{NaSCN}$ & NI & 0.0162 & & 0.084 & \\
\hline $\mathrm{KBr}$ & 0.083 & & & & 0.071 \\
\hline KI & 0.29 & & & & 0.055 \\
\hline $\mathrm{K}_{2} \mathrm{CO}_{3}$ & & 0.012 & & & \\
\hline $\mathrm{NH}_{4} \mathrm{SCN}$ & NI & 0.008 & & & \\
\hline $\mathrm{NH}_{4} \mathrm{OOCCH}_{3}$ & NI & & NI & & \\
\hline$\left(\mathrm{CH}_{3}\right)_{4} \mathrm{NBr}$ & NI & 0.014 & 0.19 & & \\
\hline$\left(\mathrm{CH}_{3}\right)_{4} \mathrm{NOOCCH}_{3}$ & 0.125 & & $\mathrm{NI}^{b}$ & & \\
\hline $\mathrm{MgCl}_{2}$ & & 0.016 & & & \\
\hline $\mathrm{Ca}\left(\mathrm{NO}_{3}\right)_{2}$ & 0.04 & 0.017 & & & \\
\hline
\end{tabular}

with all tested inhibiting coalescence relative to the pure solvent. These observations necessarily lead to a reassessment of our understanding of bubble coalescence inhibition in aqueous electrolyte solutions.

We see electrolyte inhibition of bubble coalescence in all solvents tested. The importance of this finding should not be underestimated. The mechanism by which electrolytes inhibit coalescence is unknown, and nonobvious, even in water. The interrelated steps are, first, the drainage of liquid to form a film and, second, the breakage of the film. The stability of short-lived foams is determined by the former process and long-lived foams by the latter process. ${ }^{42}$ Therefore the stability of short-lived foams produced by electrolytes should be governed by the film thinning process and more viscous solutions should produce more stable foams. Surfactants influence film thinning primarily by altering the hydrodynamic boundary condition from that of a slip condition to no-slip, which greatly decreases the rate of drainage in a thin film. We have previously suggested that electrolytes may also be able to alter the hydrodynamic boundary condition. ${ }^{25}$ Solution properties such as surface tension and ion gradient will affect the film drainage, and we hypothesized that the differences among solvents might lead to large differences in bubble coalescence behavior.

All solvents showed some propensity for thin films to be stabilized by electrolyte over the collision lifetime of our system. All showed this trend to be dependent upon salt concentration over a narrow, and similar, range. This is an indicator that electrolyte inhibition of bubble coalescence is a universal dynamic effect, rather than a change in surface forces or physical properties, which vary widely between the solvents tested. It is very unlikely that any surface force is manifest so similarly between these

(42) Bergeron, V. An introduction to forces and structures in individual foam and emulsion films. In Foams and Emulsions; Sadoc, J. F., Rivier, N., Eds.; Kluwer Academic: Dordrecht, 1999; p 45. 


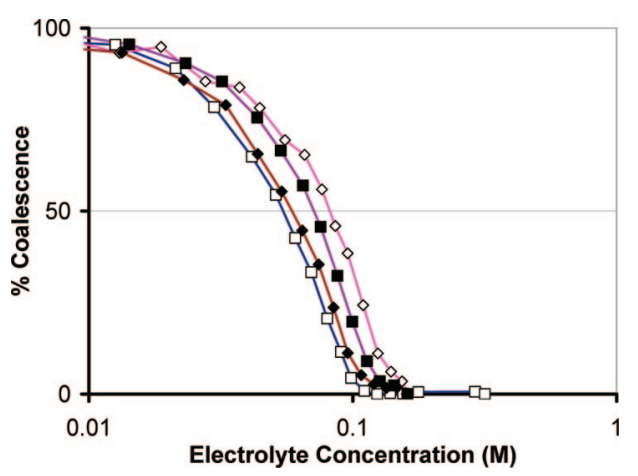

Figure 5. Inhibition of bubble coalescence by electrolytes in DMSO solutions plotted against log of concentration. $\mathrm{KI}(\square), \mathrm{NaBr}(\diamond), \mathrm{KBr}$ $(\square), \mathrm{NaClO}_{4}(\diamond) .100 \%$ coalescence is defined in pure DMSO; $0 \%$ coalescence is a stable, low, voltage signal in inhibiting electrolytes.

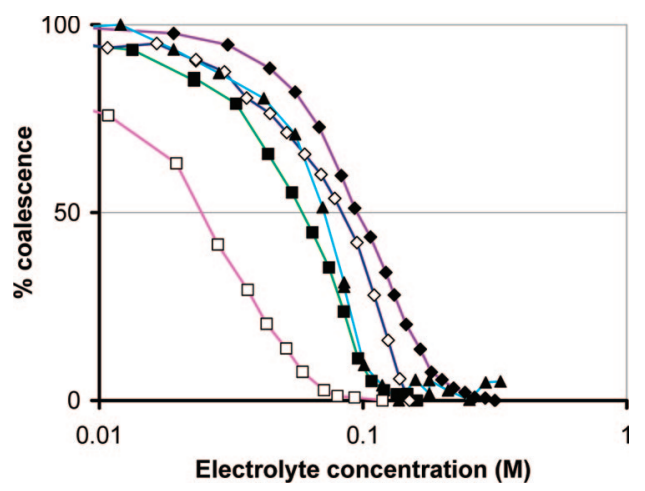

Figure 6. Comparison of typical monovalent salt coalescence inhibition in each solvent tested, as a function of log of electrolyte concentration. $\mathrm{NaClO}_{4}$ in methanol $(\square), \mathrm{NaBr}$ in DMSO $(\square), \mathrm{LiCl}$ in formamide $(\boldsymbol{\Delta})$, $\mathrm{NaSCN}$ in propylene carbonate $(\diamond), \mathrm{NaCl}$ in $\mathrm{H}_{2} \mathrm{O}(\diamond) .100 \%$ coalescence is defined in the pure solvent in each case; $0 \%$ coalescence is a stable, low, voltage signal in inhibiting electrolytes.

solvents and the short lifetime of these transient foams suggests that the thinning process rather than surface forces controls the stability.

We have not measured physical properties of nonaqueous solutions. However, we can compare the "power" (the concentration required to produce an inhibitory effect) of inhibiting salts in different solvents and look for any correlation with physical properties. Figure 6 shows coalescence vs concentration curves for typical inhibiting electrolytes in each solvent. Comparisons in specific salts can also be made in some cases from the transition concentrations in Table 5, above; however solubility and reactivity constraints prevented use of any common electrolyte in all the solvents, and the transition concentrations can be affected by even small amounts of contaminant (particularly, water), and therefore they may be slightly underestimated.

Overall we observe that the concentration of typical inhibiting electrolyte required for a given coalescence level, increases in the order: methanol $<$ DMSO $<$ formamide $<$ propylene carbonate $<\mathrm{H}_{2} \mathrm{O}$. That is, bubbles in methanol are most strongly stabilized by salt. This sequence agrees with none of the solvent physical properties in Table 2.

Ion Specificity. Ion-specific electrolyte effects are observed in formamide and propylene carbonate as well as in water. In propylene carbonate the ion assignments to different classes vary from those in water, in particular the anions $\mathrm{SCN}^{-}$and $\mathrm{ClO}_{4}{ }^{-}$ fall into the " $\alpha$ " category alongside $\mathrm{Cl}^{-}$and $\mathrm{Br}^{-}$, whereas in water they behave differently and are classified " $\beta$ " anions. In formamide the same assignments as those made for water apply across the salts tested. It is difficult to make a case as to why formamide should behave more like water than any of the other solvents tested.

In both solvents the distinction between salt classes is less clear than in water. It may make more sense to distinguish salts by their relative strength as inhibitors; "inhibiting" vs "lessinhibiting" rather than the description previously used to describe the latter class in water, as having "no effect". This is particularly so in light of other studies ${ }^{43,44}$ and recent observations in our laboratory, suggesting that "non-inhibiting" salts may inhibit coalescence at concentrations above $0.5 \mathrm{M}$. We note that in all these studies the contribution of contaminants has not been ruled out as the source of bubble coalescence inhibition at very high concentrations.

There is no obvious link between ion-specific electrolyte inhibition of bubble coalescence and bulk solvent properties. Formamide, like water, is a protic solvent but so is methanol, and these behave very differently. Propylene carbonate and methanol have hydrophobic groups but again these solvents demonstrate different electrolyte effects. The phenomenon of ion-specific behavior is observed in most high-salt systems and is hotly studied and debated. No universal basis or mechanism has been found. The "combining rules" found in the water-vapor system are not observed in other systems that exhibit ion specificity, such as biological systems. It is, therefore, significant in itself that we observe analogous behaviors in other solvents.

We have previously proposed that in aqueous systems bubble coalescence is related to the arrangement of ions at the interface and the subsequent changes to microscopic structure. Further, it was suggested that interfacial ion arrangement can be understood in terms of the combining rules. ${ }^{45}$ In this model $\alpha$ cations and $\beta$ anions are present at the water-vapor interface, while $\beta$ cations and $\alpha$ anions are withdrawn from the water - vapor interface. In our previous publication, ${ }^{25}$ we showed that the results in water of bubble coalescence inhibition by single and mixed electrolytes are consistent with the hypothesis of ion separation in the interfacial region, though the ion assignments are not always in agreement with simulation and spectroscopic studies. This lack of agreement (generally observed across experimental techniques and simulation studies) perhaps reflects the difficulties in probing a mobile interface with Ångstrom-level depth resolution. We can compare our coalescence inhibition results in nonaqueous solutions to available data on interfacial ion positioning studied using the same techniques of computer dynamics simulations and surface-selective investigative techniques such as vibrational sum frequency generation, second harmonic generation, and ion scattering. ${ }^{35}$ Of the solvents tested here, research using such techniques has been done for methanol and formamide.

In molecular dynamics simulations of aqueous $\mathrm{NaI}$, the $\mathrm{I}^{-}$ shows a distinct preference for the water-vapor interface, while $\mathrm{Na}^{+}$exists subsurface. Dang ${ }^{46}$ reports that, in methanolic NaI, the iodide is also stable at the interface and will inhabit the interface more than sodium ion, so there is some ion separation. However the preference relative to the bulk is not particularly strong. It is suggested that iodide subsurface positioning is driven by the ordering of interfacial methanol, with hydrophobic methyl groups at the surface and the hydroxyl group toward the bulk liquid. ${ }^{35}$ This contrasts with water which shows "dangling $\mathrm{OH}$

(43) Hofmeier, U.; Yaminsky, V. V.; Christenson, H. K. J. Colloid Interface Sci. 1995, 174, 199.

(44) Christenson, H. K.; Bowen, R. E.; Carlton, J. A.; Denne, J. R. M.; Lu, Y. J. Phys. Chem. C 2008, 112, 794.

(45) Marcelja, S. Curr. Opin. Colloid Interface Sci. 2004, 9, 165.

(46) Dang, L. X. J. Phys. Chem. A 2004, 108, 9014. 
groups" exposed to the vapor phase. Höfft et al. ${ }^{47,48}$ also compared aqueous and methanolic solution interfaces, using simulations as well as metastable impact electron spectroscopy and ultraviolet photoelectron spectroscopy to study CsI and CsF at the surface of amorphous solid water and methanol. The iodide segregation at the water-vapor interface is not observed in methanol, but they found that iodide lies closer to the surface than do $\mathrm{Na}^{+}$and $\mathrm{Cs}^{+}$. We note that this study (consistent with other work on aqueous interfaces ${ }^{29,31,49}$ ) found no surface preference of the fluoride ion for either solvent interface; however, sodium fluoride is found to inhibit bubble coalescence in water. Ultimately, it may be necessary to determine the precise arrangements of ions in the interfacial region in order to resolve the discrepancies between different experimental techniques as different techniques may probe different depths of the interface.

Recent work by Andersson and co-workers ${ }^{50,51}$ uses molecular dynamics simulations and neutral impact collision ion scattering spectroscopy (NICISS) to look at the formamide/vapor interface, with salts NaI, LiI, and LiCl. A distinct enhancement of iodide at the interface was found, using ion backscattering. Chloride shows some surface enhancement, lower than iodide. The enhancement of iodide at the interface is less than that observed for equivalent concentrations in water, according to NICISS experiments and to simulations. In both solvents the cation (lithium or sodium) shows no surface enhancement, and solution surface tension increase indicates overall depletion of electrolytes from the surface region, despite the iodide positioning at the interface. These results suggest that ion interfacial positioning in formamide is qualitatively similar to positioning in $\mathrm{H}_{2} \mathrm{O}$. That is, the halides show some surface preference increasing for more

(47) Höfft, O.; Borodin, A.; Kahnert, U.; Kempter, V.; Dang, L. X.; Jungwirth, P. J. Phys. Chem. B 2006, 110, 11971.

(48) Höfft, O.; Kahnert, U.; Bahr, S.; Kempter, V. J. Phys. Chem. B 2006, 110, 17115 .

(49) Jungwirth, P.; Tobias, D. J. J. Phys. Chem. B 2001, 105, 10468.

(50) Andersson, G.; Krebs, T.; Morgner, H. Phys. Chem. Chem. Phys. 2005, 7, 2948.

(51) Andersson, G.; Morgner, H.; Cwiklik, L.; Jungwirth, P. J. Phys. Chem. C 2007, 111, 4379 . polarizable anions, and the alkali metal ions show no surface propensity relative to the bulk.

On the basis of this data, if there is a connection between interfacial ions and bubble coalescence, we might expect the electrolyte effect to be similar in $\mathrm{H}_{2} \mathrm{O}$ and formamide and less so in methanol. Our results are consistent with this prediction, but any link between ion separation at the interface, and coalescence inhibition, is far from clear. Methanol demonstrates little ion separation but a high degree of coalescence inhibition, and at the formamide interface, only inhibiting salts have been investigated by simulation and spectroscopic studies.

\section{Conclusions}

Electrolytes can inhibit bubble coalescence in nonaqueous systems. Coalescence inhibition was observed in all solvents tested: methanol, formamide, propylene carbonate, and DMSO. Coalescence inhibition in formamide and propylene carbonate shows ion-specificity. As in water, electrolytes can be categorized as "inhibiting" or "less-inhibiting", depending on the concentration required to elicit particular coalescence. Furthermore, we note that ion-combining rules are not unique to the water-gas system. Formamide shows cation and anion assignments consistent with those in water, while propylene carbonate assignments are analogous but different.

The ability of electrolytes to inhibit bubble coalescence is not confined to aqueous systems but rather seems to be a general property. This strongly suggests that the mechanism of coalescence inhibition is related to the film thinning process rather than any surface force which should vary dramatically between solvents. We suggest that this is most likely due to a reduction in film drainage rate caused by a change from a slip to a no-slip boundary condition. The particular arrangement of ions in the interfacial region is thought to mediate the boundary condition.

Acknowledgment. V.S.J.C. gratefully acknowledges financial support from the Australian Research Council.

LA8008738 